\title{
Aberrant Forms of Mycobacterium phlei Produced by Streptomycin and their Multiplication on Streptomycin-free Media
}

\author{
By S. E. JUHASZ \\ Department of Bacteriology, McGill University, and Royal Edward Laurentian \\ Hospital, Montreal, Quebec, Canada
}

(Received 29 September 1961)

\begin{abstract}
SUMMARY
Aberrant forms in a streptomycin-sensitive strain of Mycobacterium phlei could regularly be shown to arise in the presence of low concentrations of streptomycin. The effect of streptomycin on the morphology of $M$. phle $i$ is comparable with that of penicillin on the morphology of organisms belonging to the Enterobacteriaceae. The transfer of aberrant forms of $M$. phlei to a streptomycin-free medium resulted in the atypical multiplication of these forms.
\end{abstract}

\section{INTRODUCTION}

It has long been suspected that atypical bizarre forms of mycobacteria similar to those known in other bacterial genera arise under adverse conditions (e.g. Metchnikoff, 1888; Mafucci, 1892; Kirchner, 1931; Kuhn, 1932). This is in constrast to the view maintained by other bacteriologists (e.g. Kahn, 1929) who maintain that mycobacteria undergo a spontaneous life cycle. According to Kahn the spontaneous life cycle of Mycobacterium tuberculosis (strain $\mathrm{H}_{37}$ ) as studied in living preparations consists of the following stages: ' $(a)$ the initial segmenting of the rod into three or more ovoid units; $(b)$ division of these units into diplococcoid forms; $(c)$ the subsequent grouping and reduction of these elements to a mass of fine, dust-like particles, from which extremely small and delicate rods were found to sprout; and $(d)$ the later development of these rods into the mature tubercle bacillus'. In order to shed more light on this controversial issue, the effect of streptomycin was studied. Experiments initially performed with a BCG strain of $\boldsymbol{M}$. tuberculosis were unsuccessful. Each concentration of streptomycin used almost immediately interrupted the multiplication of BCG without bringing any change into their morphology. On the other hand, $M$. phlei proved to be very appropriate for a study of the morphological changes, the origin and the further fate of aberrant forms of mycobacteria on exposure to streptomycin.

\section{METHODS}

A streptomycin-sensitive Mycobacterium phlei strain from the culture collection of the Department of Bacteriology, McGill University, was used in the present study. Mutants resistant to $100-1000 \mu \mathrm{g}$. streptomycin $/ \mathrm{ml}$. were obtained from this sensitive strain of $M$. phlei by serial transfer to Tryptose agar media containing increasing concentrations of streptomycin and were used for control purposes. Blocks were cut from Difco Tryptose agar base plates which contained 0.25-0.5 $\mu \mathrm{g}$. 
streptomycin/ml., which inhibits the normal growth of this organism. The behaviour of rods not yet irreversibly damaged by streptomycin was studied, on the other hand, by transfer from a streptomycin medium to an otherwise identical but streptomycin-free medium. The multiplication of untreated streptomycin-sensitive M. phlei rods was studied on streptomycin-free Tryptose agar blocks, while the reproduction of streptomycin-resistant $M$. phlei organisms was observed on Tryptose agar blocks containing $100 \mu \mathrm{g}$. streptomycin $/ \mathrm{ml}$. The blocks in either case were inoculated with a minute amount of $M$. phlei in order to have only a few rods in the microscope field. After they were covered with a coverslip and sealed with paraffin, they were kept in a stage incubator at $37^{\circ}$ or at room temperature (about $\mathbf{2 5}^{\circ}$ ). Microphotographs were taker at selected intervals with the Zeiss photomicroscope 'Microphot' on ADOX KB 14 films.

\section{RESULTS}

Normal development in the absence of streptomycin

Series 1 (Pl. '1, figs. 1-8) shows the typical multiplication of streptomycinsensitive organisms of Mycobacterium phlei on a streptomycin-free medium. All organisms underwent simple transverse fission in the course of these observations. The generation time, as can be directly read from the micrographs, varied between 4 and $6 \mathrm{hr}$.

\section{Multiplication of streptomycin-resistant organisms}

Series 2 (Pl. 1, figs. 9-17) served for comparison. Streptomycin-resistant organisms of Mycobacterium phlei were grown in $100 \mu \mathrm{g}$. streptomycin $/ \mathrm{ml}$. Their growth and reproduction was entirely comparable with that of streptomycinsensitive organisms grown in absence of streptomycin. Similarly, they multiplied by simple transverse fission, streptomycin did not affect in the least their shape, and the generation time found with them was also $4-6 \mathrm{hr}$.

\section{The effect of streptomycin}

Several trials were necessary to determine the amount $(0.25-0.5 \mu \mathrm{g} . / \mathrm{ml}$.) of streptomycin required to inhibit conventional binary fission of Mycobacterium phlei but to allow survival in an altered form, and even multiplication, when the action of streptomycin was suspended at the right time. Serial micrographs are presented here to show the effect of low concentrations of streptomycin. It should be noted that organisms behave very individually under the effect of streptomycin.

Series 3 (Pl. 2). Two of four rods remained unchanged. The rod at the left top and that at the right bottom elongated and then split into two. Both developed granules inside the rod and then became club-shaped and vacuolized.

Series 4 (Pl. 3). The single rod at the right side divided once more after exposure to streptomycin. The left one of the pair thus arising developed a club at the top which then became vacuolized. The right one became granulated. In a small group of bacteria 4 out of 5 organisms began to elongate very soon. Two at the top divided and became granulated. After 2 days' exposure, the lysis and disintegration of rods started. Granules embedded in amorphous masses, products of the fusion of disintegrating rods, were to be seen at the end of this process. 


\section{Multiplication of aberrant forms on streptomycin-free medium}

Two series of micrographs on Pl. 4 show the development of organisms which, after previous exposure to streptomycin, were inoculated into streptomycin-free medium. One of the most interesting features of this development was that although these organisms were removed from the streptomycin medium on which they were previously cultured, the effect of streptomycin did not stop immediately. On the contrary, it sometimes became more accentuated. Also, it could be very clearly shown that not only the exposure to streptomycin had a different effect on each organism, but also had the removal of streptomycin. Thus the individuality of organisms, the different patterns of behaviour of rods of the same descent already studied by Hughes (1955) with penicillin was once more confirmed.

Series 5 (Pl. 4, Figs. 36-41). This is a $3 \frac{1}{2}$ day observation. The preparation was kept at $37^{\circ}$ during this period. Out of four rods that appeared in the field, one was lysed in about $\mathbf{2 4} \mathrm{hr}$. after transfer to a streptomycin-free medium. The lysis of the cell wall liberated intracellular granules which now appeared as free granules in the medium. The upper one of two parallel rods did not show any morphological change. The lower one elongated and developed clubs at both ends. Neither of them showed any further sign of viability. On the contrary, the single rod on the right of this group of bacteria elongated, formed a club at the right pole and fragmented into coccobacillary forms. Later, as it can be seen, the coccobacillary forms subdivided into even smaller coccoid forms. The club-shaped other end elongated and divided first into two cocci and one rod, and after subsequent fissions into a chain of five cocci of different sizes.

Series 6 (Pl. 4, Figs. 42-47). The rod, the development of which will be shown here, was found adjacent to the previous group of bacteria. This organism underwent a very similar development to that shown with the previously described rods. After incubation for $24 \mathrm{hr}$. on a streptomycin-free medium, it elongated and developed a club at the left pole and by fragmentation it threw off a small rod at the other end. Overnight, this small rod subdivided into a group of smaller rods and coccobacillary forms, while the central part remained unchanged. One cannot be sure whether the club-shaped part released two cocci and thus emptied, or whether after elongation it fragmented into two and later into three coccoids. Such coccoids, if transferred again to streptomycin-free media, can elongate into rods and form, after several divisions, microcolonies consisting entirely of rods. Without further transfer, however, the coccoids, as could be seen, divided like cocci and the microcolonies thus formed consisted of coccoids.

\section{DISCUSSION}

The effect of streptomycin on the cytology of Mycobacterium phlei resembles in many respects that of penicillin. Both affect the division of bacteria; there is an increase in the protoplasmic material, while division becomes inhibited; the rods elongate and become granulated. Under the effect of both penicillin and streptomycin, the individuality of organisms becomes more pronounced than it appears under favourable conditions of growth. On the other hand, there is a marked difference between the two agents concerning their persistent effect after the removal of 
bacteria from the drug-containing medium. While the lysis of some organisms may continue after the removal of penicillin, with streptomycin even intact rods may start to lyse after the removal of this agent. Spheroplasts and L-forms that can be provoked by the action of penicillin (Vadasz \& Juhasz, 1955 a; Vadasz \& Juhasz, $1955 b$; Juhasz, Lovas \& Egyessy, 1955; Matman, Tunstall, Matthews \& Gordon, 1960) could not be detected with streptomycin. The reversion to typical rods will occur in both cases through atypical modes of multiplication, like fragmentation, extrusion, budding or sprouting. In the first stage these atypical modes of multiplication will result in the appearance of intermediate fungus- or amoeba-like forms. Only later, as a result of several such multiplications do they arrive at a stage where the known bacterial forms reappear and assume the typical mode of reproduction: simple transverse fission. There is a much bigger latency time after the removal of streptomycin than after the removal of penicillin. Furthermore, the aberrant forms produced by streptomycin do not start multiplying immediately after the removal of streptomycin, as do the penicillin-spheroplasts and L-forms after removal of penicillin. Some of the aberrant forms undergo further destructive changes (like lysis or possibly sudden death without morphological changes), even those forms which show at a later time reversion to the bacterial forms, first elongate and occasionally form clubs (Series 5 and 6 on Pl. 4).

It can still be concluded that streptomycin has an effect on the cytology of mycobacteria similar to that of penicillin on bacteria of the Enterobacteriaceae. All the findings reported here refer to Mycobacterium phlei and not to any of the types of $M$. tuberculosis. Still, the fact that actually similar forms could be observed in $M$. tuberculosis (Juhasz \& Mankiewicz, 1958) but could not be traced back directly to the typical forms shows the significance of such models. From a previous study it was concluded that aberrant forms arise spontaneously, at least without the introduction into the medium of any deleterious agent. This holds true even in the light of new facts and is supported by recent findings on mycobacterial L-forms (Matman et al. 1960). While abnormal forms can arise spontaneously, this happens only rarely and at random; thus it is hard to follow the origin and further development. On the other hand, streptomycin increases the frequency of the appearance of abnormal forms and the regular occurrence of the process provoked by streptomycin makes it possible to obtain details of the morphological transformation of typical rods into aberrant forms.

This work was supported by a National Research Council of Canada grant and by the Harrison-Watson scholarship of McGill University. The author wishes to thank Dr Edith Mankiewicz and Professor R. W. Reed for helpfully critical discussions; he also gratefully acknowledges the supply of streptomycin by Messrs Merck, Sharp and Dohme, Montreal.

\section{REFERENCES}

HuGHEs, W. H. (1955). The differences in antibiotic sensitivity of closely related single cells of Proteus vulgaris. J. gen. Microbiol. 12, 269.

Juhasz, I., Lovas, B. \& Egyessy, D. M. (1955). Electron micrographic examination of bacteriogenesis from filtrable forms (Salmonella enteritidis var. Danysz). Acta physiol. hung. 8, 97.

Juhasz, S. E. \& Mankiewicz, E. (1958). Atypical forms of tubercle bacilli. Nature, Lond. 181, 929. 
Journal of General Microbiology, Vol. 28, No. 1

Plate 1
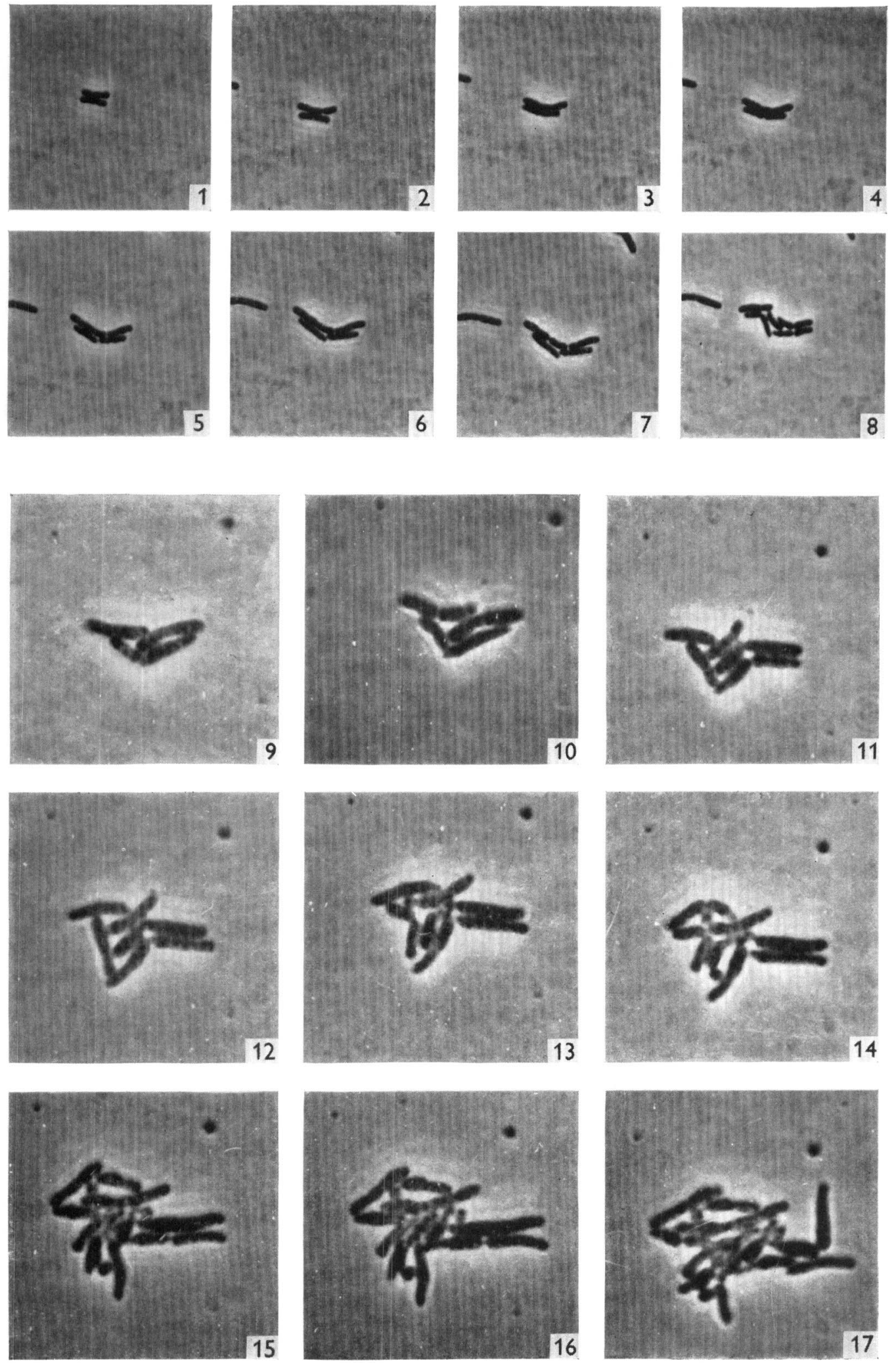

S. E. JUHAS\%

(racing p. 1:) 

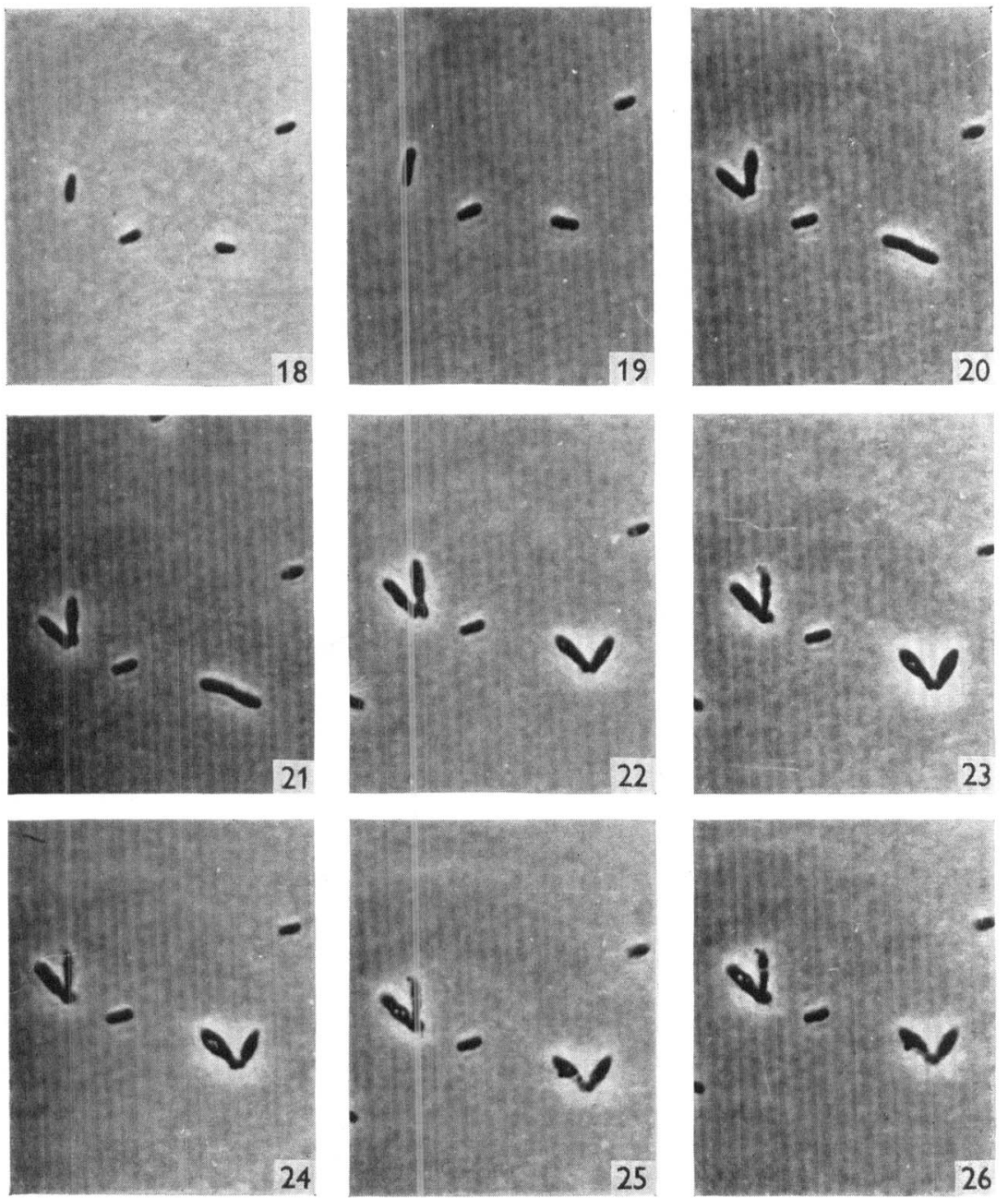

S. W. JUIIAS\% 

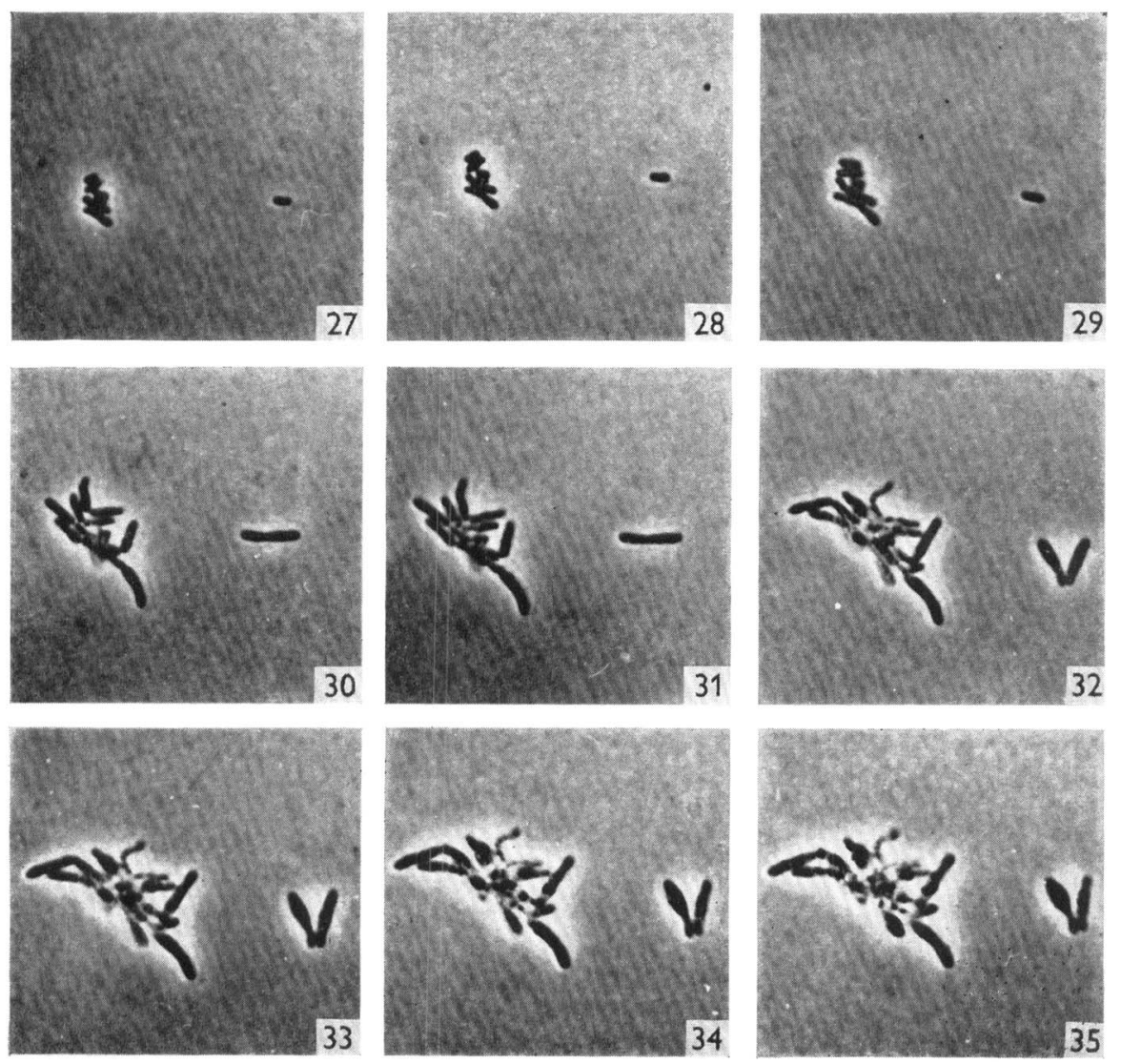
Journal of General Microbiology, Vol. 28, No. 1

Plate 4
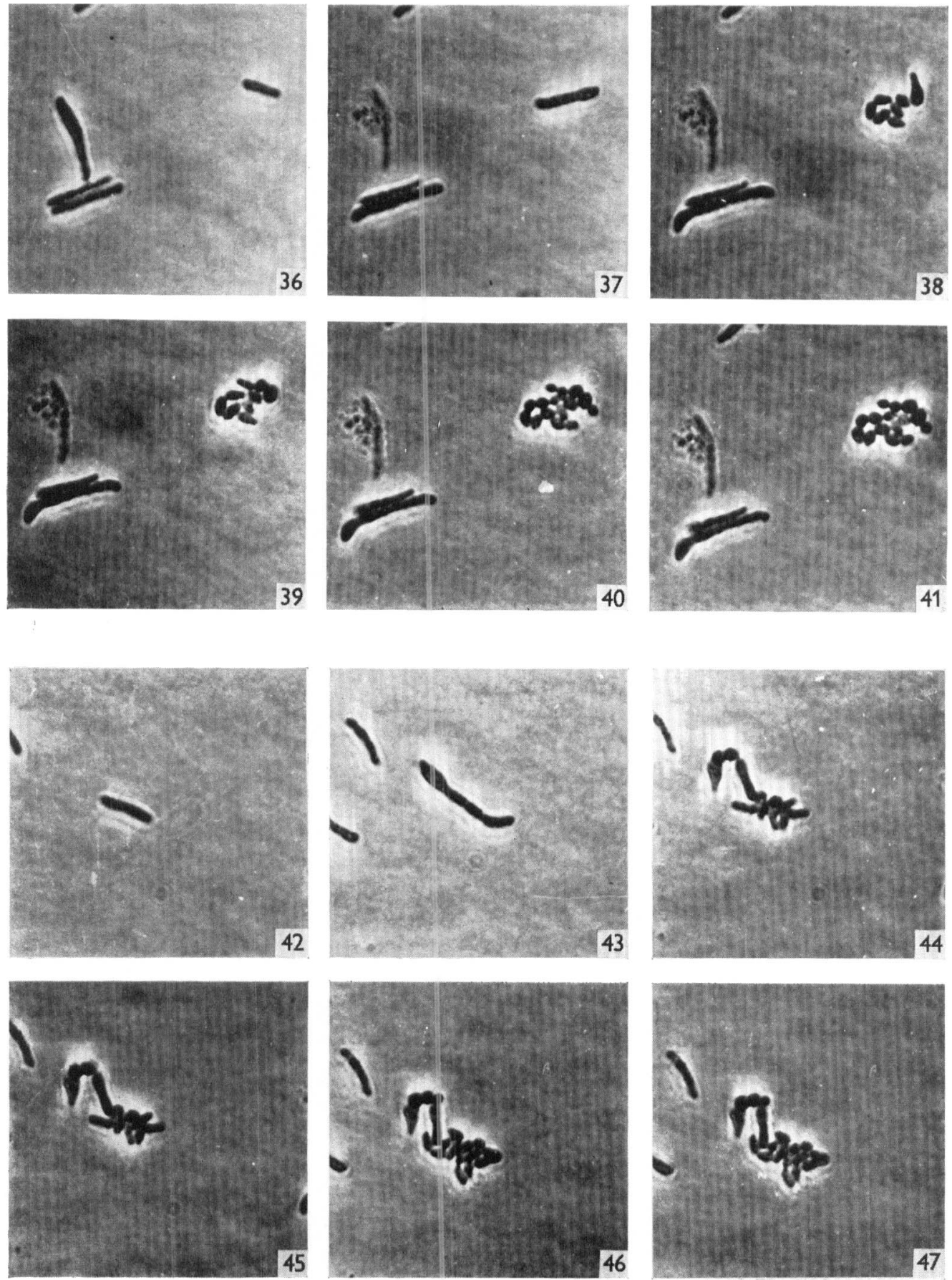

S. E. JUHASZ 
KAHN, M. C. (1929). A developmental cycle of the tubercle bacillus as revealed by singlecell studies. Amer. Rev. Tuberc. 20, 151.

Krrchner, O. (1931). Zur Veränderlichkeit des Tuberkuloseerregers in morphologischer Hinsicht. Beitr. Klin. Tuberk. 77, 72.

Kuhn, P. (1932). Über die C-Form des Tuberkelbazillus. Z. Immun.Forsch. 74, 93.

Mafucci, A. (1892). Die Hühnertuberkulose. Z. Hyg. Infekt.-Kr. 11, 445.

Matman, L. H., Tunstall, L. H., MatThews, W. W. \& Gondon, D. L. (1960). L-variation in Mycobacteria. Amer. Rev. Resp. Dis. 82, 202.

Meтchnikoff, E. (1888). Ueber die phagocytäre Rolle der Tuberkelriesenzellen. Virchows Arch. 113, 63.

Vadasz, J. \& Juhasz, I. (1955 $a, b)$. Plasma globules arising from Salmonella enteritidis under the influence of penicillin and their reversion to the original bacillary forms. Nature, Lond. 176, 168 (preliminary report) and Acta biol. hung. 6, 171.

\section{EXPLANATION OF PLATES}

Plate 1

(a) Simple transverse fission of streptomycin-sensitive Mycobacterium phlei on a streptomycin-free medium. An $8 \frac{1}{2} \mathrm{hr}$. process. The preparation was kept at $37^{\circ}$. Magnification, $\times 1600$. Time of exposure: fig. $1=0 \mathrm{hr}$., fig. $2=1 \frac{1}{2} \mathrm{hr}$, fig. $3=2 \frac{1}{2} \mathrm{hr}$., fig. $4=4 \mathrm{hr}$, fig. $5=5 \mathrm{hr}$., fig. $6=6 \mathrm{hr}$, fig. $7=7 \frac{1}{2}$ hr., fig. $8=8 \frac{1}{2} \mathrm{hr}$.

(b) Simple transverse fission of streptomycin-resistant $M$. phlei on Tryptose agar containing $100 \mu \mathrm{g}$. streptomycin $/ \mathrm{ml}$. An $11 \mathrm{hr}$. process. Preparation was kept at 37 ${ }^{\circ}$. Magnification, $\times 2700$. Time of exposure: fig. $9=0 \mathrm{hr}$, fig. $10=1 \frac{1}{2} \mathrm{hr}$., fig. $11=3 \frac{1}{2} \mathrm{hr}$., fig. $12=5 \mathrm{hr}$, fig. $13=6 \frac{1}{2} \mathrm{hr}$., fig. $14=7 \mathrm{hr}$, fig. $15=9 \mathrm{hr}$., fig. $16=10 \mathrm{hr}$., fig. $17=11 \mathrm{hr}$.

\section{Plates 2 and 3}

The effect of $0.25 \mu \mathrm{g}$. streptomycin $/ \mathrm{ml}$. on the morphology and division of $M$. phlei. A 7 -day process. The preparations were kept at $37^{\circ}$ during the day and at room temperature overnight. Magnification: Pl. $2, \times 1650$. Time of exposure: fig. $18=0 \mathrm{hr}$., fig. $19=8 \frac{1}{2} \mathrm{hr}$., fig. $20=29 \mathrm{hr}$., fig. $21=32 \frac{1}{2}$ hr., fig. $22=54 \frac{1}{2}$ hr., fig. $23=103 \frac{1}{2} \mathrm{hr}$, fig. $24=126 \frac{1}{2} \mathrm{hr}$., fig. $25=143 \mathrm{hr}$, fig. $26=167 \mathrm{hr}$. $(7$ days $)$. Magnification: Pl. $3, \times 1400$. Time of exposure: fig. $27=0 \mathrm{hr}$., fig. $28=$ $4 \frac{1}{2}$ hr., fig. $29=8 \frac{1}{2}$ hr., fig. $30=29$ hr., fig. $31=32 \frac{1}{2}$ hr., fig. $32=54 \frac{1}{2}$ hr., fig. $33=71 \frac{1}{2}$ hr., fig. $34=76 \frac{1}{2}$ hr., fig. $35=82$ hr. ( $3 \frac{1}{2}$ days $)$.

Plate: 4

Changes in the morphology and multiplication of $M . p h l e i$ when transferred after a 24 hr. exposure to $0.5 \mu \mathrm{g}$. streptomycin $/ \mathrm{ml}$. to a streptomycin-free medium. A $3 \frac{1}{2}$ day process. Preparations were kept at $37^{\circ}$ day and night. Magnification, $\times 2000$. Time of exposure: fig. $36=0 \mathrm{hr}$., fig. $37=24 \mathrm{hr}$., fig. $38=40 \mathrm{hr}$., fig. $39=50 \mathrm{hr}$., fig. $40=70 \mathrm{hr}$., fig. $41=118 \mathrm{hr}$. (5 days). Fig. $42=0 \mathrm{hr}$, fig. $43=24 \mathrm{hr}$, fig. $44=40 \mathrm{hr}$, fig. $45=50 \mathrm{hr}$, fig. $46=70 \mathrm{hr}$., fig. $47=118 \mathrm{hr}$. $(5$ days $)$. 